\title{
Inheritance of Allergic Diseases
}

\author{
Bow Vet Maciej Kucharski* \\ Department of International Medicine, Poland \\ *Corresponding author: Bow Vet Maciej Kucharski, Department of International Medicine, Smocza street 24/14, 01-034 Warsaw, Poland
}

Submission: 監 February 21, 2018; Published: 㘹 May 09, 2018

\begin{abstract}
This paper describes some of the mechanisms of gene regulation and their impact on the development of a topic dermatitis. Characterized main factors affecting the appearance of the disease in susceptible individuals. Described the role of stress in the immune system disorder. It shows the relationship between mitochondrial genome and the risk of development of allergic inflammation.
\end{abstract}

Keywords: Atopy; Astma; Inheritance; Mitochondrium; Alergic diseases

\section{Introduction}

When the sperm joins the egg cell, the zygote forms. To her, each cell carries half the genetic material. The developing embryo and then the mature organism is therefore diploid. The divine organism is similar to its parents. This feature has been used by man since he managed to domesticate and farm animals. But the continual development of cities, agricultural culture, changes in the behaviour of people and, consequently, of animals does not affect their health and functioning in the surrounding world. Constant genetic progress, the development of civilization, influences the genome, the processes that take place in it. The affected organism is trying to adapt to the various factors and give it to the offspring in its genetic material. Genome "all the time" is active, it is 'moving'.

The mechanisms underlying gene expression regulation described below in the course of allergic diseases are not without significance. Why is this happening, the carrier does not get sick? Why alleles inherited from the mother are conducive to the development of allergic reactions in her children? These are the questions that My People is just trying to answer and it will be many years before we know the secret.

\section{DNA, histons?}

DNA in the cell nucleus is wound on the so-called. Histone proteins This complex is called chromatin and its basic structural unit is the nucleus. Chromatin is present in the cell in two forms: Euchromatine and Heterochromatine. Such a structure (loose or clustered chromatin) allows you to read the information contained therein in a variety of ways. The molecular mechanisms that affect chromatin modifications include: DNA methylation, his tone protein modification, chromatin remodelling or non-coding effects. RNA interference. Modification of histone proteins (phosphorylation, histones acetylation) leads to changes in chromatin structure, which affects gene expression or blockage.

\section{Epigenetics}

It is one of the divisions of genetics, which deals with the study of changes in gene expression, which are inherited by mitotic or meiotic inheritance but are not related to changes in DNA sequences. As an example, modifications of the chromatin structure in the nucleus of the so-called $\mathrm{CpG}$ islands can be reported here.

These islands are usually within the genome within the gene promoter. Methylation of $\mathrm{CpG}$ islands within the promoter sequence disables the 'gene'. The process occurs within cytosine on both strands of DNA. The methylation pattern is preserved in a process called semi- conservative replication. After replication, the old thread is methylated and the new one is not. Methyl transferees attaches to the new DNA strand (the CpG islands) with methylmicin.

It has been found that the heritable pattern of methylation (gene imprinting, genomic stigmatization) occurs in the course of atopic dermatitis in humans. Gene imprinting in short is based on the methylation of the chromatin regions necessary for the single, allelic expression of the stigma gene. Interestingly, the parental genomic stigma established during game to genesis is inherited by the off spring and is not altered during personal development [1]. Reprogramming of genes at early embryonic development is also known, where DNA methylation is actively altered. Here as an example of an epileptic gene regulation, it is possible to cite the fact that in women exposed to a potential allergen during the early gestation period, their offspring may already have symptoms of allergy to these allergens [2]. Atopic dermatitis is a hereditary predisposition to an over-inflammatory reaction caused by contact 
with an allergen. Allergen gets into the body with food, inhaled air, through the skin, etc.

The clinical symptom of this intensified reaction is pruritus. Consequences are the wounds resulting from it (complicated by pathogens), chronic medications including in steroid preparations, antibiotics, etc. More information the reader will find in my articles [3-9]. It is known that this disease is multi-factorial. Many genes affect its appearance in the sensitive organism, but environmental factors are not without significance in the development of this disease. It is not possible to present a simple inheritance model when the disease is so complex [10-15].

On the one hand, genetic factors (for example, the genes involved in atopic pathogenesis) genes for cytokines, their receptors, growth factors, Non-specific immune-TLR2, TLR9, CARD15, CD14,chemokine genes - RANTES, eotaxin,- Transcriptional factors - STAT6, IRF2, PHF11,Hormone receptor genes $\beta 2$ adrenergic receptor,- genes encoding structural proteins of the epidermisfilaggrin, chymase, chymotrypsin, SPINK5, glutathione transferase, caspase activating agents, -IFN- $\gamma$, IL-4, IL-4R $\alpha$, IL-9, IL-10, IL-13, Lymphofotaxin alpha, NOS1, PAF, TGF- $\beta 1$, TLR4, TNF- $\alpha$, On the other hand, there are environmental factors: Allergens, the chemistry of the surrounding environment, excessive, appropriately chemicals in the so-called homes. Hygienic hypothesis- from isolation from infectious agents - by using strong hygienic agents or antibiotics as a 'medicine for everything'.

Complementary flora in the body affects the proper functioning of T lymphocytes. Changes in the composition of the bacterial floracaused, among others. By lack of stimulation of TLR receptors (Toll Like Receptor) by microorganisms completely harmless to humans, somehow impair the proper functioning of $\mathrm{T}$ regulating $\mathrm{T}$ cells. The main feature of these cells / tasks is to "decide" whether to trigger an allergen response or not. $\mathrm{T}$ lymphocytes reg. They also participate in an important process, among others. When to lead to the 'extinction' of so-called. An outbreak of inflammation.

\section{On the third hand family factors}

In the course of a topy both in humans and in animals, it has been observed that, in related individuals, this disease appeared more frequently than unrelated [16-22]. It is worth noting that the clinical symptoms of atopy in monozygotic twins are not consistent. Monozygotic twins arise from one zygote that divides into two cells during the first 14 days of pregnancy.

In monozygotic twins (when both parents are ill), the chances of having atopic dermatitis are about $80 \%$, but in twins, about $23 \%$. The risk of developing this condition when both parents are ill is around $15 \%$. But when a mother suffers, the risk of developing the disease in her child is $57 \%$, and if her father suffers from a disease, it is $46 \%$.The multi factorial nature of this disease, the mutations in the genes, make it sometimes possible to find people whose parents suffer from atopic dermatitis and they do not. It is known that this disease can be caused by a mutation in the various alleles of a given gene in a patient (genetic heterogeneity).
Otherwise, in patients predisposed to the disease (carriers) there is no clinical symptoms. In genetics such a situation is referred to as incomplete penetration (penetration) of the gene. The classical definition tells us that this is the frequency with which the dominant or recessive gene / heterozygous system will manifest itself in the phenotype of the host. Total penetration is when individuals with the same genotype have the same phenotype and incomplete penetration occurs when not all individuals with the same genotype.

\section{They reveal their characteristic phenotype}

In the course of genetic testing, atopic dermatitis in humans, it was found that only allele inherited from one parent (gene imprinting) was present in the patients. Why is this unknown? A certain light casts a mechanism of inheritance of the mitochondria after the mother, and the effect of mutation in mitochondrial genes on the functioning of cells in susceptible individuals.

\section{Disorders in the mitochondrion?}

Mitochondria are the energy centres of the cell, participate in cellular respiration, regulate the membrane potential, process apoptosis, etc. There are many organelles in this cell. The genome of these organelles is spherical. Mitochondria are inherited only after mother. It was found that dysfunctions in the mitochondria may be one of the factors predisposing to the development of allergic disease in susceptible individuals. It is about a SNP (polymorphism of single nucleotides in the mitochondrial genome).

During the study of polymorphisms in the mitochondrial genome, haplo groups have been identified that have been associated with IgE levels, but also with the development of an atopic phenotype. This relationship has also been demonstrated in the case of the inheritance of these SNPs by the mother (sperm mitochondria are not introduced into the cytoplasm of the egg during fertilization) and thus predisposing to the development of atopy in susceptible individuals.

\section{Fenokopy and atopic dermatitis?}

The term phenoxy refers to the "qualities" developed by the influence of environmental factors on the body. It is not a 'hereditary' trait, but by the association of individuals in which it is revealed, it may develop into a hereditary trait. In the study of atopic dermatitis in humans, stress is linked to the pathogenesis of atopy. Stress is one of the factors responsible for exacerbation of atopic dermatitis. Chronic stress is affecting the nervous system. It manifests itself in two ways: on the skin is the perception of pruritus stimuli and on the other hand it affects the immune system and mast cells. It has been found that the nervous system may affect the stimulatory or inhibitory immune system.

Investigating the link between stress and atopic dermatitis in humans, many surprising facts have been found. Patients with dermatitis have elevated levels of anxiety and depression compared to healthy people. Parents of children with atopic dermatitis were characterized by emotional anxiety and guilt or even overdose 
for their sick children. The exact impact of the environment on the mental state of people with atopic dermatitis is unknown. It is known that chronic stress can aggravate the condition of the disease by exacerbating it. Patients suffering from various skin diseases, among others. Atopic dermatitis was characterized by a stronger physiological response to stress. With this situation, the mechanism of action of neurotransmitters such as P, VIP, should also be linked. These neurotransmitters may exhibit stimulating or inhibitory effects on immune cells. Substance P stimulates mast cells to release inflammatory mediators, which affect the nerve endings, leading to pruritus.

Broadly in filtrated inflammatory cell in filtration in the inflammatory foci that occurs in the skin, and especially the cytokines produced by these cells, may increase the sensitivity of the nerve endings to stimulation and thereby exacerbate the symptoms of pruritus. This causes these hypersensitive nerve endings to be stimulated, stimulated by inflammatory peptides. Neuropeptides substance P or VIP, which intensify itching in the skin, are released in the further stage and consequently deepens clinical symptoms on the skin. And here comes the question of whether a dog, living next to a man who is similarly influenced by him, can develop similar stress mechanisms? And exacerbation of the symptoms of the disease is related to chronic stress? Can anxiety, depression, or other stressful conditions affect the dog's nervous system and immune system response to pruritus?

The stress response plays a sympathetic system and hypothalamic-pituitary-adrenal axis. As a result of triggering a stressful stimulus, adreanaline and nor adrenaline are excreted, glucose is mobilized (the source of energy). The body adjusts to fighting or escape. After its disappearance the organism returns to equilibrium. But what about when the stress of a dog is constantly exposed? In this case the hypothalamic-pituitary-adrenal axis is activated.

The adrenal cortex begins to synthesize glucocorticoids, among others. Cortisol This hormone remains in the blood until a few days after the stressor is usually around 2-4 days, after which it is removed from the body. But when exposure to stress is permanent and it can be, for example, when they are too rare to walk with their guardian, long-term stay of the dog at home, lack of contact with other animals, aggressive behavior in his surroundings, quarrels and fights in the owner's house, etc. This leads to constant secretion, among others Cortisol. The previous one after the previous ejection has not yet been removed from the body. Chronic stress develops. It affects not only the entire body of the dog, but also the immune system, reducing its proper functioning. Can a sensitive individual be one of the factors predisposing to the development of a topy-this question is out of the question?

\section{References}

1. Marta Olszewska, Maciej Kurpisz, Postepy Hig Med Dosw (2010) Methylation and its regulatory role towards the genomic parental stigma 64: 642-649.

2. Miwa Shinohara, Wakiguchi H, Saito H, Matsumoto K (2007) Symptoms of allergic rhinitis in women during early pregnancy are associated with higher prevalence of allergic rhinitis in their offspring., Allergol Int 56(4): 411-417.

3. (2011) Kucharski Maciej food allergies.

4. (2011) Kucharski Maciej Selected cytokines in atopic dermatitis in dogs.

5. (2011) Kucharski Maciej Cat Flea and allergic flea dermatitis.

6. (2011) Kucharski Maciej Infection of Staphylococcus sp. As a complication of atopic dermatitis in dogs.Veterinary Warehouse 20: 164.

7. (2010) Kucharski Maciej Mushroom allergens in atopic dermatitis in dogs.

8. (2015) Kucharski Maciej, Selected cell mechanisms for atopic dermatitis in dogs.

9. (2015) Genetic base of atopic dermatitis in dogs. Veterinary.

10. Marsella R, Thierry Olivry (2003) Animal models of atopic dermatitis. Clinics in Dermatology 21(2): 122-133.

11. Merryman-Simpson AE, Wood SH, Fretwell N, Jones PG, McLaren WM, et al. (2008) Gene (mRMA) expression in canine atopic dermatitis microarray analysis. Vet Dermatol 19(2): 59-66.

12. Wollenberg A, Kraft S, Oppel T, Bieber T (2000) Atopic dermatitis: pathogenic mechanisms. Clinical and Experimental Dermatology 25(7): 530-534.

13. Sinke JD, Rutten VP, Willemse T (2002) Immune dysregulation in atopic dermatitis. Vet Immunol Immunopathol 87(3-4):351-356.

14. Baker BS (2006) The role of microorganisms in atopic dermatitis. Clin Exp Immunol 144(1): 1-9.

15. Tashkun I (2006) Histopathological changes of skin in allergic diseases in dogs. Veterinary Life 81 (8).

16. Becker K G (1999) Comparative genetics of type 1 diabetes and autoimmune disease:Common loci, common pathways? Diabetes 48(7): 1353-1358.

17. Woronowicz W (2008) Use of DNA microarray for allergy testing. Biotechnology 4 (83): 115-130.

18. Chial H (2008) Mt DNA and mitochondrial diseases. Nature Education 1 (1): 217

19. Bartoszek B (2010) Atopic dermatitis (AZS) as a psychosomatic disease. Research analysis, Studies in Psychology, KUL 16: 57-74.

20. Łapińska J (2014) Effects of dog stress - how to prevent them. Vetbechativism 2

21. Saavedra-Molina, Alexander Kurosky, Boldogh I, Aguilera-Aguirre L, Bacsi A, et al. (2009) Mitochondrial Dysfunction Increases Allergic Airway Inflammation J Immunol 183(8): 5379-5387.

22. Benjamin Rabby A, Klanderman B, Murphy A, Mazza S, Camargo CA, et al. (2007) A common mitochondrial haplogroup is associated with elevated total serum IgE levels. J Allergy Clin Immunol 120(2): 351-358. 

International License

For possible submissions Click Here

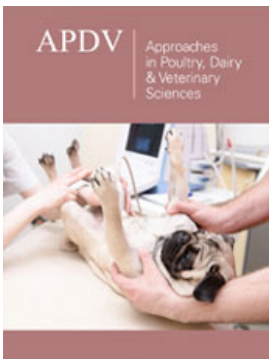

Approaches in Poultry, Dairy \& Veterinary Sciences

\section{Benefits of Publishing with us}

- High-level peer review and editorial services

- Freely accessible online immediately upon publication

- Authors retain the copyright to their work

- Licensing it under a Creative Commons license

- Visibility through different online platforms 